\title{
Diagnostic single nucleotide polymorphism markers to identify hybridization between dromedary and Bactrian camels
}

\author{
Emily Ruiz • Elmira Mohandesan • Robert R. Fitak • \\ Pamela A. Burger
}

Received: 9 October 2014/ Accepted: 7 January 2015/Published online: 13 January 2015

(C) The Author(s) 2015. This article is published with open access at Springerlink.com

\begin{abstract}
The technique to produce hybrid Tulu or Nar camels from crosses between dromedary and Bactrian camels is common throughout Middle Eastern and Central Asian countries. Formerly, these hybrids were highly valued as strong and persistent pack animals but today are bred to improve milk or wool quality in the respective species and for camel wrestling. We developed a diagnostic single nucleotide polymorphism (SNP) panel to identify cryptic ancestry in $\mathrm{F}_{1}$ hybrids and their backcrosses by selecting loci from whole genome data, which were fixed for different alleles in either dromedary or domestic and wild Bactrian camel. With this SNP panel we are able to identify the hybridization patterns in camels with uncertain origins, support hybrid breeding management and to detect potential rare dromedary introgression in the last wild Bactrian camels in Mongolia and China.
\end{abstract}

Keywords Single nucleotide polymorphisms (SNPs) · Hybrid camel · Next generation sequencing (NGS) · Camelus bactrianus · Camelus dromedarius · Introgression

\section{Technical note}

Anthropogenic hybridization between livestock populations or between domestic species and their wild relatives has been practiced since the early phases of domestication.

Electronic supplementary material The online version of this article (doi:10.1007/s12686-015-0420-z) contains supplementary material, which is available to authorized users.

E. Ruiz · E. Mohandesan · R. R. Fitak · P. A. Burger $(\bowtie)$

Institut für Populationsgenetik, Vetmeduni Vienna,

Veterinärplatz 1, 1210 Vienna, Austria

e-mail: pamela.burger@vetmeduni.ac.at
In Old World camelids, hybridization between Bactrian camels (Camelus bactrianus) and dromedaries (Camelus dromedarius) was associated with the transportation of goods along the multiple routes of the Silk Road. This practice intended to produce animals with the robustness of the Bactrian camel, the endurance of dromedary, and the ability to tolerate sharply contrasting climatic conditions (Wilson 1984). Today, hybridization facilitates improved milk and wool yield in hybrid Tulu or Nar camels from Middle Eastern and Central Asian countries. Commonly, two hybridizing methods are recognized, Kurt-nar (dromedary female $\times$ Bactrian male) and Kez-nar (Bactrian female $\times$ dromedary male) followed by $F_{1}$ backcrossing with either dromedary for increased milk productivity or Bactrian camel for wool and cold resistance (Faye and Konuspayeva 2012). $F_{2}$ hybrids $\left(F_{1} \times F_{1}\right)$ in Old World camels are usually not favoured because of a difficult character and weak progeny performance (Faye and Konuspayeva 2012). In western regions of Turkey, a relished sport is camel wrestling, where prized male Tulus compete against each other in heavily regulated fights (Vedat 2010). In this study, we developed diagnostic single nucleotide polymorphisms (SNPs) to identify $F_{1}$ hybrids between dromedary and Bactrian camels and their backcrosses. These markers will facilitate the validation of noninvasive or historic samples with unclear or otherwise cryptic ancestry.

As part of an on-going research project, we sequenced the genomes of 25 Old World camelids [C. dromedarius $(\mathrm{n}=9)$, C. ferus $(\mathrm{n}=9), C$. bactrianus $(\mathrm{n}=7)]$ and aligned them to the $C$. ferus CB1 genome assembly (Genbank ID: GCA_000311805.2). To verify their species identity, these source individuals had previously been genotyped at 18 nuclear microsatellite loci and Sanger sequenced for $800 \mathrm{bp}$ of mitochondrial DNA (unpublished 
data). We identified SNPs according to the guidelines presented by Van der Auwera et al. (2013) and selected polymorphisms that were fixed for different alleles between $C$. dromedarius and both Bactrian camel species, C. bactrianus and C. ferus (see 'Supplementary Methods' in Online Resource 1). We designed PCR primers around each SNP using PRIMER3 (Untergasser et al. 2012) and selected 26 primer pairs (Online Resource 2) for validation.

We tested the 26 candidate loci using PCR and Sanger sequencing (see 'Supplementary Methods' in Online Resource 1) on a panel of control individuals including three Bactrian camels, three dromedaries, two hybrids $\left(\mathrm{F}_{1}\right.$ and $\mathrm{F}_{1}$ backcross) and a two-humped individual with uncertain genetic background. All 26 candidate loci produced PCR products of the expected sizes, but we selected for sequencing only those 12 loci which produced single strong bands over consistent conditions (Table 1). Upon sequencing, all 12 loci were diagnostic between dromedaries and Bactrian camels (Table 2). However, in the $\mathrm{F}_{1}$ hybrid (Hyb56) only 10 (83.3\%) of the diagnostic loci were heterozygous and the remaining two loci were homozygous for the Bactrian allele (Table 2). This suggests that at these two loci (HP264 and HP288) the Bactrian allele may be present at low frequency in dromedaries as a result of being a shared ancestral polymorphism (not diagnostic) or from historical hybridization between the two species. The $\mathrm{F}_{1}$ backcross (Hyb55) was heterozygous for two $(16.7 \%)$ of the diagnostic loci and homozygous for the Bactrian allele in the remaining loci. In the twohumped individual with uncertain origin (DC575), four $(33.3 \%)$ of the diagnostic loci were heterozygous, demonstrating hybrid ancestry in this animal (Table 2).

With this panel of diagnostic SNPs it is possible to identify $F_{1}$ hybrids between dromedary and Bactrian camels and to differentiate $F_{1}$ backcrosses to either parental species. Thus, we can validate the status of individuals with unclear parental origins in regions where the two species are extensively crossbred (e.g. Kazakhstan, Turkey). Because we selected short PCR fragments $(\leq 150 \mathrm{bp})$ the panel can be applied in non-invasive, historical and archaeological (ancient) samples, where the morphology of the preserved materials cannot retrieve unambiguous classification (Gallik et al. 2015). Moreover, with this marker set we will be able to detect potential, rare dromedary introgression in Bactrian camel populations in Mongolia and China. As hybridization between domestic Bactrian camels and their wild relatives has been demonstrated (Silbermayr and Burger 2012; Silbermayr 2009), the threat for dromedary introgression into the last wild two-humped camels (C. ferus) in the Mongolian Gobi (Yadamsuren et al. 2012) and Chinese Taklimakan and Lop Nor deserts (Lei et al. 2012) is small but existing. Therefore, this primer set is an important contribution to the conservation of the genetic integrity of this critically endangered species.

Table 1 Primer sequences and information for the 12 diagnostic loci

\begin{tabular}{|c|c|c|c|c|c|c|}
\hline Locus & Primer sequence $\left(5^{\prime}-3^{\prime}\right)^{\mathrm{a}}$ & Scaffold $^{\mathrm{b}}$ & Position $^{\mathrm{b}}$ & Alleles & $\mathrm{T}^{\mathrm{m}}$ & Length \\
\hline HP206 & TGTCAGACTGTTAGGCATTGC CATCCAAGTCTCCATCTAACCC & NW_006210212.1 & 501206 & $\mathrm{C} / \mathrm{G}$ & 57 & 125 \\
\hline HP379 & AGGATGCCATCATGTCAGG GAGGGAGCTCTCATGAATAGG & NW_006210464.1 & 1151379 & G/A & 58 & 150 \\
\hline HP405 & CCAGGAGCTTTTCGAGTAGC CAGCACAGAGAACTCACTGC & NW_006211106.1 & 5030405 & $\mathrm{G} / \mathrm{C}$ & 59 & 125 \\
\hline HP429 & GCAGGCATACAAACTAACCC GCTTTTCTTTCTGGCTCAGG & NW_006210666.1 & 2288429 & $\mathrm{~A} / \mathrm{C}$ & 57 & 125 \\
\hline HP458 & TGTGACCAGACAGACCAAGG TGTGGCTTAGGGTCTTTATGG & NW_006210457.1 & 11458 & $\mathrm{~T} / \mathrm{C}$ & 58 & 140 \\
\hline HP501 & GAATAGATTGGGGAGCAAGC CTCTTCTCCATCCCTATGGC & NW_006211169.1 & 218501 & $\mathrm{~A} / \mathrm{T}$ & 59 & 125 \\
\hline HP597 & ATGAACAGTTTGGGTTTGGG CGCGATGTCACCTTTATAGG & NW_006211126.1 & 6065597 & $\mathrm{~A} / \mathrm{C}$ & 59 & 125 \\
\hline HP633 & GCATGTAGAAGGTTTGCATAGG CAGCCTTTCTTGCATCTGG & NW_006210489.1 & 4279633 & $\mathrm{G} / \mathrm{C}$ & 57 & 125 \\
\hline HP900 & CCACATGCTCAGGTATCTGG GGGATTCCTTGTGCTACAGC & NW_006211075.1 & 294900 & $\mathrm{G} / \mathrm{C}$ & 59 & 125 \\
\hline HP930 & CTCCCAGGAAACAAAAGTCC TTTGGGAGTGTTCTGTCTGC & NW_006210745.1 & 3459930 & $\mathrm{C} / \mathrm{A}$ & 59 & 125 \\
\hline HP264 & TGGACAGAAACTTTGTGTCTCC TTTGGTAAGGGCATGAATCC & NW_006211022.1 & 519264 & $\mathrm{~T} / \mathrm{C}$ & 59 & 125 \\
\hline HP288 & GTCTATGAGGGCGTTTCTGC CAGCCTTCTTGTTCTGTTCG & NW_006211252.1 & 214288 & T/A & 59 & 125 \\
\hline
\end{tabular}

See Online Resource 2 for additional details on these and the remaining 14 primers examined

$\mathrm{T}_{\mathrm{m}}$ : primer annealing temperature

Length: PCR product length

${ }^{\text {a }}$ Left (forward) primer given above the right (reverse) primer

b Accession number from GenBank and position of the SNP in the scaffold 


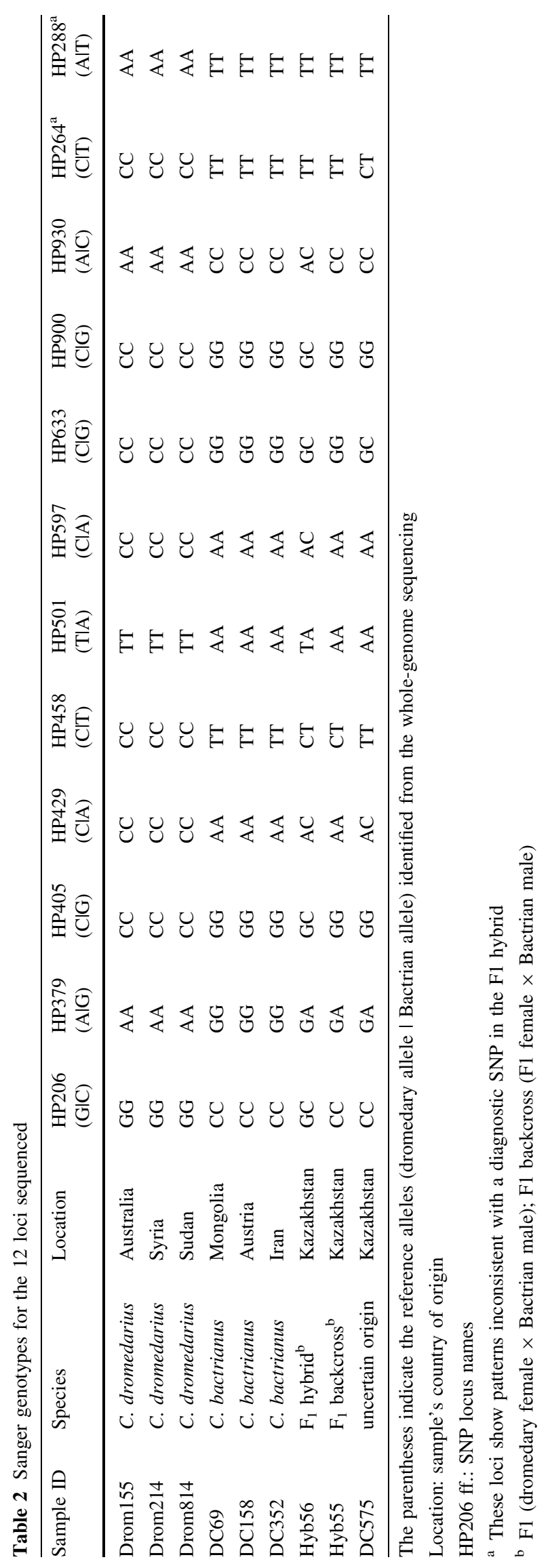


Acknowledgments We are grateful to C. Schlötterer for hosting the project. Funds were granted by the Austrian Science Foundation FWF (P24706-B25) to P. Burger, who is recipient of an APART fellowship (11506) from the Austrian Academy of Sciences.

Open Access This article is distributed under the terms of the Creative Commons Attribution License which permits any use, distribution, and reproduction in any medium, provided the original author(s) and the source are credited.

\section{References}

Faye B, Konuspayeva G (2012) The encounter between Bactrian and dromedary camels in Central Asia. In: Knoll EM, Burger PA (eds) Camels in Asia and North Africa. Interdisciplinary perspectives on their significance in past and present. Austrian Academy of Sciences, Vienna, pp 29-36

Gallik A, Mohandesan E, Schulz UM, Krenn M, Forstenpointner G, Burger PA (2015) A sunken ship of the desert at the river Danube in Tulln, Austria. PLoS One (PONE-D-14-47954)

Lei Y, Hare H, Guoying Y, Yun C (2012) The status of the wild camel in China. In: Knoll EM, Burger PA (eds) Camels in Asia and North Africa. Interdisciplinary perspectives on their significance in past and present. Austrian Academy of Sciences, Vienna, pp 55-60

Silbermayr K (2009) The detection of hybridization on the Mongolian wild Bactrian (Camelus ferus) using molecular markers. Doctoral Thesis, Veterinärmedizinische Universität, Wien

Silbermayr K, Burger PA (2012) Hybridization: a threat to the genetic distinctiveness of the last wild old world camel species. In: Knoll EM, Burger PA (eds) Camels in Asia and North Africa. Interdisciplinary perspectives on their significance in past and present. Austrian Academy of Sciences, Vienna, pp 69-76

Untergasser A, Cutcutache I, Koressaar T, Ye J, Faircloth BC, Remm M, Rozen SG (2012) Primer3-new capabilities and interfaces. Nucl Acid Res 40:e115

Van der Auwera GA, Carneiro MO et al (2013) From FastQ data to highconfidence variant calls: the genome analysis toolkit best practices pipeline. Cur Protoc Bioinform 43:11:11.10:11.10.1-11.10.33

Vedat Ç (2010) Examining cultural tourism attractions for foreign visitors: the case of camel wrestling in Selcuk Ephesus. Turizam $14: 22-40$

Wilson RT (1984) The camel. Longman, London

Yadamsuren A, Dulamtseren E, Reading RP (2012) The conservation status and management of wild camels in Mongolia. In: Knoll EM, Burger PA (eds) Camels in Asia and North Africa. Interdisciplinary perspectives on their significance in past and present. Austrian Academy of Sciences, Vienna, pp 45-54 